\title{
AN INEQUALITY FOR THE DISTRIBUTION OF THE BROWNIAN GRADIENT FUNCTION ${ }^{1}$
}

\author{
BURGESS DAVIS
}

ABstract. The Brownian gradient function of a harmonic function $u$ in the unit disc is shown to be distributionally about as large as the classical area function of $u$. This distribution function inequality strengthens some integral inequalities of Burkholder and Gundy.

If $u$ is a harmonic function on the open unit disc $D$ the classical area and nontangental maximal functions are defined to be, respectively,

and

$$
A_{\sigma}(u)(x)=\left[u(0)^{2}+\int_{\Gamma_{\sigma}(x)}|\operatorname{grad} u|^{2}\right]^{1 / 2}
$$

$$
N_{\sigma}(u)(x)=\sup \left\{|u(z)|: z \in \Gamma_{\sigma}(x)\right\},
$$

where $\Gamma_{\sigma}(x)$ is the interior of the smallest convex region containing the disc of radius $\sigma<1$ and the point $x=e^{i \theta}$. The Brownian analogues of these functions are

and

$$
S(u)=\left[u(0)^{2}+\int_{0}^{\tau}|\operatorname{grad} u(B(t))|^{2}\right]^{1 / 2}
$$

$$
u^{*}=\sup _{0 \leqq t<\tau}|u(B(t))|
$$

where $B(t)$ is two dimensional Brownian motion started at 0 and $\tau$ is the first time $|B(t)|=1$.

It is known that for a large class of functions $\Phi$, including all powers $\Phi(\lambda)=\lambda^{p}, p>0$, there are positive constants $c, C$ depending only on $\sigma$ and $\Phi$ but not on $u$ such that $\int_{0}^{2 \pi} \Phi\left(A_{\sigma}(u)\right) d x, \int_{0}^{2 \pi} \Phi\left(N_{\sigma}(u)\right) d x$, and $E \Phi(S(u))$ all lie between $c E \Phi\left(u^{*}\right)$ and $C E \Phi\left(u^{*}\right)$, that is, the four functions are $\Phi$-equivalent. See [1] and [2].

The two maximal functions have been shown to be still more strongly related in [1], where it is proved that there are positive constants $c^{\prime}$ and $C^{\prime}$

Received by the editors January 24, 1972 and, in revised form, May 16, 1972.

AMS (MOS) subject classifications (1969). Primary 3111, 6062.

Key words and phrases. Harmonic function, area function, Brownian motion, distribution inequality.

${ }^{1}$ Research under NSF Grant GP-19222.

(c) American Mathematical Society 1973 
depending only on $\sigma$ such that, for $\lambda>0$,

$$
c^{\prime} P\left(u^{*}>\lambda\right) \leqq m\left(N_{\sigma}(u)>\lambda\right) \leqq C^{\prime} P\left(u^{*}>\lambda\right) .
$$

Here the following one-sided distribution function inequality for the area function will be proved.

THEOREM 1. There is a positive constant $K$ depending only on $\sigma$ such that

$$
m\left(A_{\sigma}(u)>K \lambda\right) \leqq K P(S(u)>\lambda)
$$

is true if $\lambda$ is a positive number and $u$ is harmonic on $D$.

Inequality (2) immediately implies

$$
E \Phi\left(A_{\sigma}(u)\right) \leqq C_{\sigma, \Phi} E \Phi(S(u))
$$

for the class of functions $\Phi$ mentioned above.

The converse of (2) is easily seen to be false, for if $u(x)=x$ then $|\operatorname{grad} u|=1$ so that $A_{\sigma}(u)^{2} \leqq \pi$, while $S(u)^{2}=\tau$. Thus a converse of (2) would require $P(\tau>C)$ to be 0 for some $C>0$. However, $P(\tau>C)>0$ for all $C>0$.

The right-hand side of (1) and inequality (2) hold for harmonic functions in two variables only. Burkholder and Gundy show, in a paper in preparation, that their analogues in higher dimensions are not true.

The half-plane analogue of (2) is the following: Let $P_{x+i y}$ be the probability measure associated with a Brownian motion $B(t)$ started at $x+i y$, and let $\eta$ be the first time $B(t)$ is real. For $0<\sigma$ and $x_{0}$ real, define $R_{\sigma}\left(x_{0}\right)$ to be the cone $\left\{x+i y: y>0,\left|x-x_{0}\right|<\sigma y\right\}$. Then there is a positive constant $K^{\prime}$, depending only on $\sigma$, such that, for all $u$ harmonic in the upper halfplane and $\lambda>0$,

$$
\begin{aligned}
m\left\{x: \int_{R_{\sigma}(x)}|\operatorname{grad} u|^{2}>\right. & \left.K^{\prime} \lambda\right\} \\
& \leqq K^{\prime} \sup _{y \geqq 0} \int_{-\infty}^{\infty} P_{x+i v}\left(\int_{0}^{n}|\operatorname{grad} u(B(t))|^{2}>\lambda\right) d x .
\end{aligned}
$$

We omit the proof, since it is a natural extension of the disc result, using the techniques of [1].

I want to thank Richard F. Gundy for many helpful comments.

In the following, $C_{1}, C_{2}, \cdots$ stand for positive constants depending at most on $\sigma$. We use $\tau$ to indicate the first time a process hits the unit circle. It is assumed with no essential loss of generality that $u(0)=0$.

If $E_{y}$ denotes expectation taken with respect to the probability distribution associated with a Brownian motion $B(t)$ started at $y \in D$ and if $f$ is a 
nonnegative continuous function on $D$, let $\bar{E}_{y} f=E_{y} \int_{0}^{\tau} f(B(t)) d t$. A formula in Hunt [5, p. 309, equation (2)] states that

$$
\bar{E}_{y} f=\int_{D} g_{D}(y, z) f(z) d z,
$$

where $g_{D}$ is the Green's function for $D$. If $S_{v}$ is the disc of radius 2 and center $y$ then $S_{y} \supset D$ so, for each $z \in D$, we have

$$
g_{D}(z, y) \leqq g_{S_{y}}(z, y)=-\log (|z-y| / 2) \leqq-\log (|| z|-| y|| / 2) .
$$

Substituting this inequality in (5) we have

$$
\bar{E}_{y} f \leqq-\int_{0}^{1} r \log \left(\left|r-r_{0}\right| / 2\right) d r \int_{0}^{2 \pi} f\left(r e^{i \theta}\right) d \theta, \quad|y|=r_{0}<1 .
$$

If $|y| \leqq \frac{1}{4}$ and $R_{y}$ is the disc of radius $\frac{3}{4}$ around $y$, then $R_{y} \subset D$, so that, for $z \in R_{y}, g_{D}(z, y) \geqq g_{R_{y}}(z, y)=-\log (4|y-z| / 3)$. In particular if $|z| \leqq 4$ then $|y-z| \leqq \frac{1}{2}$ and $g_{D}(z, y) \geqq-\log \left(\frac{2}{3}\right)$, implying

$$
\bar{E}_{y} f \geqq-\int_{0}^{1 / 4} \log \left(\frac{2}{3}\right) r d r \int_{0}^{2 \pi} f\left(r e^{i \theta}\right) d \theta, \quad|y| \leqq \frac{1}{4} .
$$

LEMMA. If $\rho$ is a continuous nonnegative subharmonic function on $D$ such that $\rho(0)=1$ and if $|z| \leqq \frac{1}{4}$ then

$$
P_{z}\left(\int_{0}^{\tau} \rho(B(t)) d t \geqq C_{1}\right) \geqq C_{1} .
$$

Proof. Let

$$
g(r)=\frac{1}{2 \pi} \int_{0}^{2 \pi} \rho\left(r e^{i \theta}\right) d \theta, \quad 0<r<1, \quad g(0)=1 .
$$

Since $\rho$ is subharmonic, $g(r) \geqq \rho(0)=1,0 \leqq r<1$, and $g$ is continuous on $[0,1)$ by the bounded convergence theorem. Therefore $\hat{\rho}\left(r e^{i \theta}\right)=$ $\rho\left(r e^{i \theta}\right) / g(r)$ is a nonnegative, continuous function on $D$ such that $\hat{\rho} \leqq \rho$ and $\int_{0}^{2 \pi} \hat{\rho}\left(r e^{i \theta}\right) d \theta=1,0<r<1$. We thus have, by (6) and (7),

$$
\bar{E}_{y} \hat{\rho} \leqq C_{2}, \quad y \in D,
$$

and

$$
\bar{E}_{y} \hat{\rho} \geqq C_{3}, \quad|y| \leqq \frac{1}{4} .
$$

If $A$ is a set let $I(A)$ be the indicator function of $A$ and if $f$ is any function defined on $A$ but perhaps not elsewhere let $\int f I(A)=\int_{A} f$.

Now let $z_{0} \leqq \frac{1}{4}$. Let $\eta$ be the first time before $\tau$ that $\int_{0}^{\eta} \hat{\rho}(B(t)) d t=C_{3} / 2$. 
The strong Markov property and (8) imply

$$
\begin{aligned}
E_{z_{0}} \int_{\eta}^{\tau} \hat{\rho}(B(t)) d t I(\eta<\tau) & =E_{z_{0}}\left[\left(\bar{E}_{B(\eta)} \hat{\rho}\right) I(\eta<\tau)\right] \\
& \leqq E_{z_{0}}\left(C_{2} I(\eta<\tau)\right)=C_{2} P_{z_{0}}(\eta<\tau)
\end{aligned}
$$

Thus

$$
\begin{aligned}
C_{3} & \leqq E_{z_{0}} \hat{\rho}=E_{z_{0}}\left(\int_{0}^{\min (\eta, \tau)} \hat{\rho}(B(t)) d t\right)+E_{z_{0}}\left(\int_{\eta}^{\tau} \hat{\rho}(B(t)) d t I(\eta<\tau)\right) \\
& \leqq C_{3} / 2+C_{2} P_{z_{0}}(\eta<\tau) .
\end{aligned}
$$

Therefore

$$
P_{z_{0}}\left(\int_{0}^{\tau} \hat{\rho}(B(t)) d t \geqq C_{3} / 2\right) \geqq P_{z_{0}}(\eta<\tau) \geqq C_{3} / 2 C_{2}
$$

Since $\hat{\rho} \leqq \rho$, we may take $C_{1}=\min \left(C_{3} / 2, C_{3} / 2 C_{2}\right)$.

The hypotheses of this lemma were simplified to avoid notation. However the analogue of (5) holds for regions $R$ other than $D$ and in particular for $D(a, r)=\{z:|z-a|<r\}$. Thus a proof exactly like the proof just given can be made to show that if $\gamma$ is the first time $|B(t)-a|=r$ and if $q$ is a nonnegative subharmonic function on $D(a, r)$ then

$$
P_{\nu}\left(\int_{0}^{\gamma} q(B(t)) d t \geqq C_{1} q(a) r^{2}\right) \geqq C_{1}, \quad|y-a| \leqq r / 4 .
$$

If $\tau_{a}$ is the first time that $|B(t)|=a$ then $B\left(\tau_{a}\right)$ is uniformly distributed on the circle $\{z:|z|=a\}$ under $P_{0}$. Thus, if $D(a, r) \subset D$,

$$
\begin{aligned}
P_{0}(|B(t)-a|=r \text { for some } & t, 0 \leqq t<\tau) \\
& =P_{0}(B(t) \in D(a, r) \text { for some } t, 0 \leqq t<\tau) \\
& \geqq P_{0}\left(B\left(\tau_{a}\right) \in D(a, r)\right) \geqq 2 r / 2 \pi a \geqq r / \pi .
\end{aligned}
$$

Let $g$ stand for $|\operatorname{grad} u|^{2}$. Let $v$ be the first time $|B(t)-a|=r / 4$ and $\mu$ the first time after $v$ but before $|B(t)-a|>r$ that $\int_{v}^{\mu} g(B(t)) d t \geqq C_{1} r^{2} g(a)$. The strong Markov property, (10) and (11) imply

$$
\begin{aligned}
P_{0}\left(\int_{0}^{\tau} g(B(t)) I(D(a, r))\right. & \left.d t \geqq C_{1} r^{2} g(a)\right) \\
& \geqq E_{0}\left(P_{33(v)}(\mu<\tau) I(v<\tau)\right) \\
& \geqq E_{0}\left(C_{1} I(v<\tau)\right) \\
& =C_{1} P_{0}(v<\tau) \geqq C_{1} r / \pi .
\end{aligned}
$$

Let $P_{0}^{\theta}$ be the probability associated with conditional Brownian motion starting at 0 and conditioned by the restriction $B(\tau)=e^{i \theta}$. Conditional 
processes of this type have been discussed by Doob in [3] and [4]. The following is a conditional version of Theorem 1 and thus immediately implies it.

THEOREM 2. $P_{0}^{\theta}\left(S^{2}(u)>C_{5} A_{\sigma}^{2}\left(e^{i \theta}\right)\right)>C_{5}$.

Proof. Assume with no loss of generality that $\theta=0$. Let

$$
T_{n}=\left\{z \in \Gamma_{\sigma}(1): 1-2^{-n}<|z| \leqq 1-2^{-n+1}\right\}, \quad n \geqq 1,
$$

and let $a_{n}$ be a point of the closure of $T_{n}$ such that $g\left(a_{n}\right)=\sup \left\{g(z): z \in T_{n}\right\}$. Since for some constant $C_{6}$, and all $n$, area $T_{n} \leqq C_{6} 2^{-2 n}$, we have

$$
\int_{T_{n}} g(x) d x \leqq C_{6} g\left(a_{n}\right) 2^{-2 n}
$$

so

$$
A_{\sigma}^{2}(1)=\int_{\cup^{\infty} T_{n}} g(x) d x \leqq \sum_{1}^{\infty} C_{6} g\left(a_{n}\right) 2^{-2 n} .
$$

Thus either $\sum_{1}^{\infty} C_{6} g\left(a_{2 n}\right) 2^{-4 n}$ or $\sum_{1}^{\infty} C_{6} g\left(a_{2 n-1}\right) 2^{-4 n+2}$ is at least half of $A_{\sigma}^{2}(1)$. Suppose without loss of generality it is the former. Let $\mu_{2 k}$ be the first time before $\tau$ that

$$
\int_{0}^{\mu_{2 k}} g(B(t)) I\left(D\left(a_{2 k}, 2^{-2 k-2}\right)\right) d t=C_{1} g\left(a_{2 k}\right) 2^{-4 k-4} .
$$

If $z \in D$ then the density of $B(\tau)$ under $P_{z}$ on the unit circle is given by the Poisson kernel

$$
P\left(z, e^{i \theta}\right)=(2 \pi)^{-1}\left(\left(1-|z|^{2}\right) /\left|z-e^{i \theta}\right|^{2}\right) .
$$

This is discussed in [3]. If $y \in D\left(a_{2 k}, 2^{-2 k-2}\right)$ then, since $a_{2 k} \in T_{2 k}, y$ satisfies $|y| \geqq 1-2^{-2 k+2}$ and $|y-1| \leqq C_{7} 2^{-2 k}$, so the Poisson kernel satisfies

$$
P(y, 1) \geqq C_{8} 2^{2 k}, \quad y \in D\left(a_{2 k}, 2^{-2 k-2}\right) .
$$

Let $P_{2 k}\left(0, e^{i \theta}\right)$ be the density of $B(\tau) I\left(\mu_{2 k}<\tau\right)$ under $P_{0}$. Then since $B\left(\mu_{2 k}\right) \in D\left(a_{2 k}, 2^{-2 k-2}\right),(12),(13)$, and the strong Markov property imply

$$
\begin{aligned}
P_{2 k}(0,1) & =E_{0}\left(P\left(B\left(\mu_{2 k}\right), 1\right) I\left(\mu_{2 k}<\tau\right)\right) \\
& \geqq C_{8} 2^{2 k} P_{0}\left(\mu_{2 k}<\tau\right) \geqq C_{9} 2^{2 k} C_{1} 2^{-2 k-2} / \pi=C_{9} .
\end{aligned}
$$

Since $P(0,1)=1 / 2 \pi$, we have that

$$
P_{0}^{0}\left(\mu_{\Sigma_{k}}<\tau\right)=P_{2 k}(0,1) / P(0,1) \geqq C_{9} /(2 \pi)^{-1}=C_{10} .
$$


Now if $f=\sum_{k=1}^{\infty} C_{1} g\left(a_{2 k}\right) 2^{-4 k-4} I\left(\mu_{2 k}<\tau\right)$, we have

while

$$
\left(E_{0}^{0} f^{2}\right)^{1 / 2} \leqq \sup |f| \leqq \sum_{k=1}^{\infty} C_{1} g\left(a_{2 k}\right) 2^{-4 k-4}
$$

$$
E_{0}^{0} f=\sum_{k=1}^{\infty} g\left(a_{2 k}\right) 2^{-4 k-4} P_{0}^{0}\left(\mu_{2 k}<\tau\right) \geqq C_{10} \sum_{k=1}^{\infty} g\left(a_{2 k}\right) 2^{-4 k-4} .
$$

Thus $\left(E_{0}^{0} f^{2}\right)^{1 / 2} \leqq C_{11} E_{0}^{0} f$, which implies $P_{0}^{0}\left(f \geqq C_{12} E_{0}^{0} f\right) \geqq C_{12}$. (See Zygmund, [6, Chapter V, 8.26].)

Since the circles $D\left(a_{2 k}, 2^{-2 k-2}\right), k=1,2, \cdots$, are disjoint we have

$$
S^{2}(u) \geqq \sum_{k=1}^{\infty} \int g(B(t)) I\left(D_{a_{2 k}}, 2^{-2 k-2}\right) d t \geqq \sum_{k=1}^{\infty} C_{1} g\left(a_{2 k}\right) 2^{-4 k-4} I\left(\mu_{2 k}<\tau\right)=f,
$$

and since $A_{\sigma}^{2}(1) \leqq C_{13} E_{0}^{0} f$,

$$
P_{0}^{0}\left(S^{2}(u) \geqq C_{12} C_{13} A_{\sigma}^{2}(1)\right) \geqq C_{12} .
$$

Thus the $C_{5}$ in the statement of the theorem may be taken to be $\min \left(C_{12}, C_{12} C_{13}\right)$. The proof is complete.

\section{REFERENCES}

1. D. L. Burkholder, R. F. Gundy and M. L. Silverstein, A maximal function characterization of the class $H^{D}$, Trans. Amer. Math. Soc. 157 (1971), 137-153. MR 43 \#527.

2. D. L. Burkholder and R. F. Gundy, Distribution function inequalities for the area integral, Studia Math. (to appear).

3. J. L. Doob, Semimartingales and subharmonic functions, Trans. Amer. Math. Soc. 77 (1954), 86-121. MR 16, 269.

4. Conditional Brownian motion and the boundary limits of harmonic functions, Bull. Soc. Math. France 85 (1957), 431-458. MR 22 \#844.

5. G. A. Hunt, Some theorems concerning Brownian motion, Trans. Amer. Math. Soc. 81 (1956), 294-319. MR 18, 77.

6. A. Zygmund, Trigonometrical series, 2nd rev. ed., Cambridge Univ. Press, New York, 1959. MR 21 \#6498.

Department of Statistics, Rutgers University, New Brunswick, New JERSEY 08903 